\title{
Comparison of particle swarm optimization and genetic algorithm for multiproduct batch plant design of protein production
}

\begin{abstract}
This work deals with the problem of the optimization of multiproduct batch plant design (MBPD) found in a chemical engineering process. The aim of this work is to minimize the investment cost and find out the number and size of parallel equipment units in each stage. For this purpose, it is proposed to solve the problem in two different ways: the first way is by using particle swarm algorithms (PSA) and the second way is by genetic algorithms (GAs). This paper presents the effectiveness and performance comparison of PSA and GAs for optimal design of multiproduct batch plant. The calculation results (investment cost, number and size of equipment, computational time, CPU time and idle times in plant) obtained by GAs are better than PSA. This methodology can help the decision makers and constitutes a very promising framework for finding asset of good solutions.
\end{abstract}

Keywords: mathematical modeling, chemical engineering optimization, particle swarm algorithms, genetic algorithms, batch plant design
Volume 7 Issue 5 - 2018

\section{Youness El Hamzaoui, Juan Antonio Alvarez \\ Arellano}

Facultad de Ingeniería, Universidad Autónoma del Carmen,

Mexico

Correspondence: Youness El Hamzaoui, Facultad de Ingeniería, Universidad Autónoma del Carmen, Mexico, Tel 00521 (999)960II38,

Email eyouness@pampano.unacar.mx; yelhamzaoui77@gmail. com

Received:January 15, 20I8 | Published:September 20, 2018

\section{Introduction}

Pharmaceutical researchers and biotechnology companies are devoted to developing medicines, such as: therapeutic proteins, human insulin, vaccines for hepatitis, food grade protein, chymosin detergent enzyme, and cryophilic protease. This allows patients to live longer, heathier, and more productive. However, in recent years, there has been an increased interest development of systematic method for the design of batch process in chemicals, food products, and pharmaceutical industries. Basically, batch plants are composed of items operating in a discontinuous way, where each batch then visits a fixed number of equipment items, as required by a given synthesis sequence so called production recipe. Many works in the literature on batch process design are based on expressions that relate the batch sizes linearly with the equipment sizes. ${ }^{1-10}$ The number required of volume and size of parallel equipment units in each stage is to be determined. Nevertheless, the design of batch plants requires involving how equipment may be utilized. However you look at it the optimal design of a multiproduct batch chemical process involves the production requirement of each product and the total production time available for all products has been considered. The number and size of parallel equipment units in each stage as well as the location and size of intermediate storage are to be determined in order to minimize the investment cost.

The aim of this work was to solve the multiproduct batch plant design problem using (PSA) and (GAs), respectively. The model presented is general, it takes into account all the available options to increase the efficiency of the batch plant design: unit duplication inphase and out-phase and intermediate storage tanks.

We have found out that PSA performed effectively and gave a solution, but we would like to solve the problem more effectively, that's why we proposed to apply GAs, an intelligent problem- solving method that has demonstrated its effectiveness in solving combinatorial optimization problem, and satisfactory results have been obtained.

\section{Materials and methods}

\section{System description and experimental data}

The case study, taken from the literature, is a multiproduct batch plant for the production of proteins. ${ }^{11}$ This example is used as a test bench since it provides models describing the unit operations involved in the process. The batch plant involves eight stages for producing four recombinant proteins, on one hand, two therapeutic proteins, human insulin (A) and vaccine for hepatitis (B) and, on the other hand, a food grade protein, chymosin (C), and a detergent enzyme, cryophilic protease (D). Figure 1 is the flowsheet of the multiproduct batch plant considered in this study. All the proteins are produced as cells grow in the fermenter. It is hardly necessary to say that the number of intermediate storage tanks is an important constituent of our process: Three tanks have been selected: the first after the fermenter, the second after the first ultrafilter, and the third after the second ultrafilter.

Vaccines and protease are considered to be intracellular. The first microfilter is used to concentrate the cell suspension, which is then sent to the homogenizer for the second microfilter, which is used to remove the cell debris from the solution proteins. The first ultrafiltration step is designed to concentrate the solution in order to minimize the extractor volume. In the liquid-liquid extractor, salt concentration $(\mathrm{NaCl})$ is used as solution in order to minimize the extractor volume. In the liquid-liquid extractor, salt concentration $(\mathrm{NaCl})$ is used to first drive the product to a poly-ethylene-glycol (PEG) phase and again into an aqueous saline solution in the back extraction. The second ultrafiltration is used again to concentrate the solution. The last stage is chromatography, during which selective binding is used to better separate the product of interest from the other proteins. 


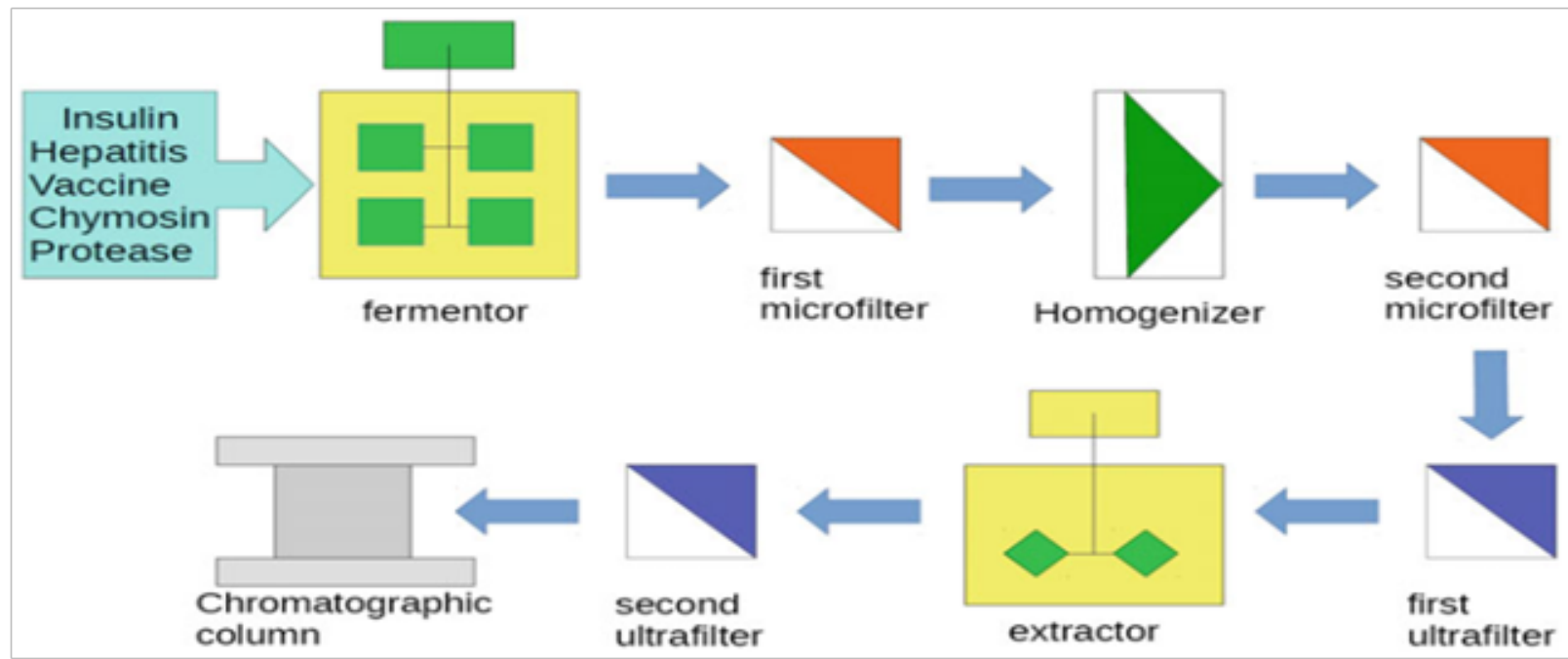

Figure I Multiproduct batch plant for protein production.

Table I Product prices and demands

\begin{tabular}{llll}
\hline Product & Name & Production (kg/year) & Price (dollar/kg) \\
\hline I & Insulin & 1500 & 8000 \\
2 & Vaccine & 1000 & 7500 \\
3 & Chymosin & 3000 & 1000 \\
4 & Protease & 6000 & 500 \\
\hline
\end{tabular}

Insulin and chymosin are extracellular products. Proteins are separated from the cells in the first microfilter, where cells and some of the supernatant liquid stay behind to reduce the amount of valuable products lost in the retentate, extra water is added to the cell suspension. The homogenizer and the second microfilter for cell debris removal are not used when the product is extracellular. Nevertheless, the first ultrafilter is necessary to concentrate the dilute solution prior to extraction. The final step of extraction, second ultrafiltration, and chromatography are common to both the extracellular and intracellular products. In Table 1 we make an estimation of production targets and product prices. ${ }^{12-14}$

\section{Problem statement}

The model formulation for DMBP's problem approach adopted in this section is based on Montagna el al. ${ }^{15}$ It considers not only Table 2 Size factors $S_{i j}(r$, retentate; $p$, permeate) treatment in batch steps, which usually appear in all types of formulation, but also represents semi continuous units that are part of the whole process (pumps, heat exchangers, etc). A semi-continuous unit is defined as a continuous unit alternating idle times and normal activity periods. Besides, this formulation takes into account midterm intermediate storage tanks, the obligatory mass balance at the intermediate storage stage, which is one of the most efficient strategies to decouple bottlenecks in batch plant design. They are just used to divide the whole process into subprocesses in order to store an amount of materials corresponding to the difference of each sub-process productivity. In this section we describe the unit models from a conceptual standpoint and also the procedure to derive the data needed for solving the mathematical model. These data are summarized in Table $2 \&$ Table 3.

\begin{tabular}{|c|c|c|c|c|c|}
\hline \multicolumn{2}{|c|}{ Stage (j) } & \multicolumn{4}{|c|}{$S_{i j}\left(\mathrm{~m}^{3} / \mathbf{k g}\right)$} \\
\hline & Unit & Insulin & Vaccine & Chymosin & Protease \\
\hline I & Fermenter & 1.25 & 0.625 & 0.415 & 0.3125 \\
\hline \multirow[t]{2}{*}{2} & \multirow{2}{*}{ Microfilter I } & $r: 1.25$ & $r: 0.625$ & $r: 0.415$ & $r: 0.3125$ \\
\hline & & $\mathrm{p}: 2.5$ & p:no & $p: 0.830$ & p:no \\
\hline 3 & Homogenizer & No & 0.155 & No & 0.08 \\
\hline \multirow{2}{*}{4} & \multirow{2}{*}{ Microfilter II } & \multirow{2}{*}{ No } & r:0.155 & No & $r: 0.08$ \\
\hline & & & $\mathrm{p}: 0.31$ & & $\mathrm{p}: 0.16$ \\
\hline 5 & Ultrafilter I & 2.5 & 0.31 & 0.83 & 0.16 \\
\hline 6 & Extractor & 0.4 & 0.2 & 0.14 & 0.1 \\
\hline 7 & Ultrafilter II & 0.4 & 0.2 & 0.14 & 0.1 \\
\hline 8 & Chromatographer & 0.05 & 0.05 & 0.05 & 0.05 \\
\hline
\end{tabular}


Table 3 Time factors $T_{i j}\left[B_{i}(\mathrm{~kg})\right]$

\begin{tabular}{llllll}
\hline Stage & & $\mathbf{T}_{i j}(\mathbf{h})$ & & & \\
\hline $\mathbf{j}$ & Unit & Insulin & Vaccine & Chymosine & Protease \\
$\mathrm{I}$ & Fermentor & 24 & 24 & 24 & 24 \\
2 & Microfilter I & $12.5 \mathrm{~A}^{-1} \mathrm{~B}_{\mathrm{i}}$ & $2.5 \mathrm{~A}^{-1} \mathrm{~B}_{\mathrm{i}}$ & $4.15 \mathrm{~A}^{-1} \mathrm{~B}_{\mathrm{i}}$ & $1.25 \mathrm{~A}^{-1} \mathrm{~B}_{\mathrm{i}}$ \\
3 & Homogeneizer & no & $0.465 \mathrm{cap}^{-1} \mathrm{~B}_{\mathrm{i}}$ & no & $0.24 \mathrm{cap}^{-1} \mathrm{~B}_{\mathrm{i}}$ \\
4 & Microfilter II & no & $3.1 \mathrm{~A}^{-1} \mathrm{~B}_{\mathrm{i}}$ & no & $1.6 \mathrm{~A}^{-1} \mathrm{~B}_{\mathrm{i}}$ \\
5 & Ultrafilter I & $105 \mathrm{~A}^{-1} \mathrm{~B}_{\mathrm{i}}$ & $5.5 \mathrm{~A}^{-1} \mathrm{~B}_{\mathrm{i}}$ & $35 \mathrm{~A}^{-1} \mathrm{~B}_{\mathrm{i}}$ & $3 \mathrm{~A}^{-1} \mathrm{~B}_{\mathrm{i}}$ \\
6 & Extractor & $I .5$ & 1.5 & 1.5 & 1.5 \\
7 & Ultrafilter II & $18 \mathrm{~A}^{-1} \mathrm{~B}_{\mathrm{i}}$ & $8 \mathrm{~A}^{-1} \mathrm{~B}_{\mathrm{i}}$ & $4.75 \mathrm{~A}^{-1} \mathrm{~B}_{\mathrm{i}}$ & $3 \mathrm{~A}^{-1} \mathrm{~B}_{\mathrm{i}}$ \\
8 & Chromatographer & 0.5 & 0.5 & 0.5 & 0.5 \\
\hline
\end{tabular}

Most of the separation processes information are taken from Asenjo and Patrick., ${ }^{16}$ the posynomial modeling approach is taken from Salomone and Iribarren., ${ }^{17}$ The general batch process literature as reported in, ${ }^{18}$ describes batch stages $j$ through a sizing equation and a cycle time that are applied for a product $i$ as follows:

$$
\mathrm{v}_{\mathrm{j}} \geq \mathrm{S}_{\mathrm{ij}} B_{\mathrm{i}}
$$

Where $V_{j}$ is the size of stage $j$, e.g., $\mathrm{m}^{3}$ of the vessel, $B_{i}$ is the batch size for product $i$, e.g., $k g$ of product exiting from the last stage, $S_{i j}$ is the size factor of stage $j$ product $i$, i.e., the size needed at stage $j$ to produce $1 \mathrm{~kg}$ of final product $i$ and $T_{i j}$ is the time required to process a batch of product $i$ in stage $j$ considering the fermentor and the insulin product as an example. If we estimate a final concentration of $50 \mathrm{~kg}$ dry biomass $/ \mathrm{m}^{3}$, that 0.4 of this biomass is proteins and 0.05 of these proteins is insulin, and an overall yield estimate of the process of 0.8 ( 0.8 of the insulin produced in the fermenter exits the chromatographic column), then the size factor for the fermenter for producing insulin can be estimate as

$$
S_{i j}=\frac{\mathrm{m}^{3}}{50 \mathrm{~kg} \times 0.4 \times 0.05 \times 0.8}=1.25 \mathrm{~m}^{3}
$$

Similarly, vaccine, chymosine, and cryophilic protease were estimated to be $0.1,0.15$, and 0.2 of total proteins of the biomass, respectively. The batch stage description is completed by estimating a processing time $T_{i j}$ for stage $j$ when producing product $i$. For the fermenter, we estimate $T_{i j}=24 \mathrm{~h}$ for all products, which includes time for charging, cell growth, and discharging.

This model of batch stages given by constraint (1a) is the simplest one. Its level of detail suffices for the fermenter and the extractor. These units are truly batch items chat hold the load to be processed and whose operations are governed by kinetics, and hence, the operating time does not depend on the batch size. The first approximation for the extractor, we take a phase ratio of (1b) for all products. Therefore, the required extractor volume is twice the inlet batch volume, while the inlet and outlet aqueous saline batches are of the same volume. It is also assumed, as a result of preliminary balances, that this operation reduces the total amount of proteins to about twice the amount of the target protein. with respect to the kinetic effects we take as first estimates ${ }^{19}$ the following times: 15 min stirring to approach phase equilibrium, $30 \mathrm{~min}$ settling to get almost complete disengaging of the phases, and $20 \mathrm{~min}$ for charging and discharging. A special consideration must be done in the case of the microfiltration, homogenization, and ultrafiltration stages. Although the mathematical model considers them batch stages, their corresponding equipment consists of holding vessels and semicontinous units that operate on the material that is recirculated into the holding vessel. The batch items are sized as described before. For example, for the homogenizer processing cryophilic protease, we estimated that the fermentor broth is concentrated 4 times up to $200 \mathrm{~kg} / \mathrm{m}^{3}$ at microfilter 1 and considered a yield of 1 because the intracellular protease is fully retained at the microfilter. Then the size factor of the homogenizer vessel is 4 times smaller than the fermenters, i.e., $S_{i j}=0.08 \mathrm{~m}^{3} / \mathrm{kg}$ protease. The sizing equation for semicontinuous items can also be found in the general batch processes literature: ${ }^{20}$

$$
R_{j}=D_{i j} \frac{B_{i}}{\grave{e}_{i j}}
$$

Where $R_{j}$ is the size of the semicontinuous item $k$, usually a rate of processing. For example, in the case of the homogenizer, it is the capacity in cubic meters of suspension per hour, but in the case of the filters $R_{j}$ is their area of filtration $\mathrm{A}_{\mathrm{j}}\left(\mathrm{m}^{2}\right) . B_{i}$ is again the batch size, $\mathrm{e}_{\mathrm{ij}}$ is the operating time that the semicontinuous item $j$ needs to process a batch of product $i$, and $D_{i j}$ is the duty factor (a size factor for semicontinuous items), i.e., the size needed at stage $j$ to process $1 \mathrm{~kg}$ of product $i$ in $1 \mathrm{~h}$. For example, if we adopt three passes through the homogenizer, its duty factor is the vessel size factor $0.08 \mathrm{~m}^{3} / \mathrm{kg} \times 3$, i.e., $D_{i j}=0.24 \mathrm{~m}^{3} / \mathrm{kg}$. The meaning of a capacity of $0.24 \mathrm{~m}^{3} / \mathrm{h}$ is that it allows $1 \mathrm{~kg}$ of final product cryophilic protease to be processed in $1 \mathrm{~h}$.

The general batch processes literature considers semicontinuous units to work in series with batch units so that their operating time are the times for filling or emptying the batch units. However, in the process considered, pumps are the only semicontinuous units, which transfer batches between the units. As the pumps cost does not have a relevant impact on the plant design, they were not explicitly modeled. The times for filling and emptying batch items were estimated and included in the batch cycle times. On the other hand, the process does have special semicontinuous units with an important economic impact on the cost. They are the homogenizer and ultrafilters, but their operating time is the batch processing time of the respective stage. The mathematical model depends on both the batch size and the size of the semicontinuous item are as follows:

$$
\begin{aligned}
& \mathrm{V}_{\mathrm{j}} \geq \mathrm{S}_{\mathrm{ij}} \mathrm{B}_{\mathrm{i}} \\
& \mathrm{T}_{\mathrm{ij}}=\mathrm{T}_{\mathrm{ij}}^{0}+\mathrm{T}_{\mathrm{ij}} \frac{B_{\mathrm{i}}}{\mathrm{R}_{\mathrm{j}}}
\end{aligned}
$$


Where $R_{j}$ refers to the size of the semicontinuous item that operates on the batch size at stage $j . \mathrm{T}_{\mathrm{ij}}{ }^{0}$ and $\mathrm{T}_{\mathrm{ij}}{ }^{1}$ are appropriate time factors that take into account contributions to the total cycle time of the stage that are either fixed amounts of time or proportional to the batch size and inversely proportional to the size of the semicontinuous item. For the homogenizer, $R_{j}$ is its capacity, $\mathrm{T}_{\mathrm{ij}}{ }^{1}$ the duty factor of the homogenizer itself, and $\mathrm{T}_{\mathrm{ij}}{ }^{0}$ includes the estimated times for filling and emptying the homogenizer holding vessel. In the case of ultrafilters, a fixed permeate flux model was considered with a rate of $20 \mathrm{~L} / \mathrm{m}^{2}$ of membrane area/h. In this case, the size of the semicontinuous item $R_{j}$ is the filtration area. $\mathrm{T}_{\mathrm{ij}}^{0}$ is again the time for filling and emptying the retentate holding vessel, and $\mathrm{T}_{\mathrm{ij}}{ }^{1}$ is the inverse of the permeate flux times the ratio $\left(\mathrm{m}^{3}\right.$ permeate $\left./ \mathrm{kg}\right)$. This ratio is estimated from a mass balance taking into account that the ultrafilters are used for a water removal from solutions up to $50 \mathrm{~g} / \mathrm{L}$ of total proteins. Ultrafilters are used to reduce the volume required at the liquid extractor and the chromatographic column. The upper bound on concentration is a constraint that avoids protein precipitation. The microfilter model is quite similar to that of the ultrafilter, but there are two batch items associated to them instead of one, the retentate and the permeate vessels, plus the semicontinuous item area of filtration. For microfilter $1 \mathrm{a}$ fixed permeate flux of $200 \mathrm{~L} / \mathrm{m}^{2} \mathrm{~h}$ is adopted. For extracellular insulin and chymosin, we estimate a total permeate (feedwater plus make up water) twice the feed, while for intracellular protease and vaccine we estimate it in $75 \%$ of the feed (the retentate is concentrated four times). For microfilter 2 a fixed permeate flux model is also used. In this case, the flux is smaller than the one in microfilter 1 because the pore size to retain cell debris is smaller than the one for whole cells. As a first estimation we take $100 \mathrm{~L} / \mathrm{m}^{2} \mathrm{~h}$ and a total permeate (feed plus make up water) twice the feed. With respect to the chromatographic column, an adsorptive type chromatography is considered, with a binding capacity of $20 \mathrm{~kg} / \mathrm{m}^{3}$ of column packing. The size factor of this unit is the inverse of that binding capacity. As a first approximation, a fixed total operating time of $0.5 \mathrm{~h}$ was estimated for loading, eluting, and washing regeneration.

Finally, the stage model is completed with a cost model that expresses the cost of each unit as a function of its size, in the form of a power law. These expressions are summarized in Table 4, with most of the cost data. ${ }^{20}$

Table 4 Cost of equipment (U.S. Dollars)

\begin{tabular}{lll}
\hline Unit & Size & Cost \\
\hline Fermenter & $V_{j}\left(m^{3}\right)$ & $63400 V^{0.6}$ \\
Micro and ultrafilters & $V_{\text {retentate }}\left(m^{3}\right)$ & $5750 V_{r}^{0.6}$ \\
Homogenizer & $V_{\text {holding }}\left(m^{3}\right)$ & $5750 V^{0.6}$ \\
& $C a p\left(m_{3} / h\right)$ & $12100 \mathrm{cap}^{0.75}$ \\
Extractor & $V_{\text {extr }}\left(m^{3}\right)$ & $23100 V^{0.65}$ \\
Chromatography & $V_{\text {chrom }}\left(m^{3}\right)$ & $360000 V^{0.995}$ \\
\hline
\end{tabular}

\section{Model equations}

The mathematical optimization model for designing the multiproduct batch plant is described in this section. The model includes the stage models described in the previous section plus additional constraints that are explained in this section. The plant consists of $M$ batch stages (in our case 8 batch stages). Each stage $j$ has a size $\mathrm{V}_{\mathrm{j}}\left(\mathrm{m}^{3}\right)$, and more than one unit can be installed in parallel. They can work either in-phase (starting operation simultaneously) or out of phase (starting times are distributed equally spaced between them). The duplication in phase is adopted in case the required stage size exceeds the specific upper bound. In this case $G_{j}$ units are selected, splitting the incoming batch into $G_{j}$ smaller batches, which are processed simultaneously by the $G_{j}$ units. After processing, the batches are added again into a unique outgoing batch. Otherwise, duplication out-of-phase is used for time-limiting stages, if a stage has the largest processing time, then it is a bottleneck for the production rate. Assigning $M_{j}$ units at this stage, working in out of phase mode, reduces the limiting processing time and thus increases the production rate of the train. For this case, the batches coming from the upstream stages are not split. Instead, successive batches produced by the upstream stage are received by different units of stage $j$, which in turn pass them at equally spaced times onto the downstream batch stage. The allocation and sizing of intermediate storage has been included in the model to get a more efficient plant design. The goal is to increase unit utilization. The insertion of a storage tank decouples the process into two subprocesses: one upstream from the tank, and the other downstream. This allows the adoption of independent batch sizes and limiting cycle times for each subprocess.

Therefore, the previously unique $B_{i}$ is changed to batch sizes $B_{i j}$ defined for product $i$ in stage $j$. Appropriate constraints adjust the batch sizes among different units. The objective is to minimize the capital cost of the plant. The decision variables in the model are as follows:

At each batch stage the number of parallel units in phase and out of phase and their size, and the installation or absence of intermediate storage between the batch stages and their size. The plant is designed to satisfy a demand of $Q_{i}(\mathrm{~kg})$ of each product $i$, for the $P$ product considered, within a time horizon $H(h)$.

In summary, the objective function to be optimized is

$$
\operatorname{MinC} \text { ost }=\sum_{j=1}^{M} M_{j} G_{j} a_{j} V_{j}^{a_{j}}+\sum_{j=1}^{M} V T_{j}^{c_{j}}
$$

Where $a_{j}$ and $\alpha_{j}, c$ and $\eta_{j}$ are appropriate cost coefficients that depend on the type of equipment being considered. $V T$ is the size of the storage tank allocated after stage $j$. The size of each unit has to be large enough to be able to process every product: 


$$
V_{j} \geq \frac{S_{i j} B_{i j}}{G_{j}} \forall i=1, \ldots, P ; \forall j=1, \ldots, M
$$

Where $S_{i j}$ is the size factor for product $i$ in stage $j$. In case of parallel units working in phase, the division of $B_{i j}$ by the number of units $G_{j}$ takes into account the reduction in the batch size to be processed by these units. The operation time $T_{i j}$ to process product $i$ at stage $j$ has the general following form:

$$
\mathrm{T}_{\mathrm{ij}}=\mathrm{T}_{\mathrm{ij}}^{0}+\mathrm{T}_{\mathrm{ij}}^{1} \frac{\mathrm{B}}{\mathrm{R}}{ }_{\mathrm{ij}} \quad \forall \mathrm{i}=1, \ldots, \mathrm{P} ; \forall \mathrm{j}=1, \ldots, \mathrm{M}
$$

Where $T_{i j}{ }^{0}$ and $T_{i j}{ }^{1}$ are appropriate constants that depend on both the product and the stage. Expression 7 accounts for a fixed and variable contribution to the total operating time. The last term in Eq 7 depends on both the batch size and the size of the semicontinuous item associated to this batch stage, as was already discussed previousely.

The limiting cycle time for product $i$ in the subprocess $h, T L^{h}$, is the largest processing time in this production train:

$$
\mathrm{TL}_{\mathrm{i}}^{\mathrm{h}} \geq \frac{\mathrm{T}_{\mathrm{ij}}}{\mathrm{M}_{\mathrm{j}}} \forall \mathrm{i}=1, \ldots, \mathrm{P} ; \forall \mathrm{j} \in \mathrm{J}_{\mathrm{j}} ; \forall \mathrm{h}
$$

Where $J$ is the set of units which conform the subprocess $h$ the division by the number of units in parallel working out of phase, $M$ takes into account the reduction in the cycle time of this stage due to the operation of $M$ units that alternatively process the consecutive batches. To avoid accumulation of material, the processing rate of both subprocess downstream and upstream of the storage tank must be the same:

$$
\left(\begin{array}{c}
B_{i}^{d} \\
T L_{i}^{d}
\end{array}\right)=\left(\begin{array}{c}
B_{i}^{u} \\
T L_{i}^{u}
\end{array}\right) \forall i=1,2, \ldots, P
$$

The constraints 9 equalizes the production rate upstream and downstream of the storage tank. To express 9 in a simple form, the inverse of the production rate of product $i\left(E_{i}\right)$, is defined as

$$
E_{i}=\frac{T L_{i}^{h}}{B_{i j}} \forall i=1,2, \ldots, P ; \forall j \in J_{h} ; \forall h
$$

Expression 10 is used to replace $T L^{n}$ in constraint 8, dropping constraint 9. The production constraint is posed as follows: during the time horizon $H$ the plant must produce the target production quantities $Q$ of each product $i$. The number of batches of each product $i$ to be produced during time $H$ is $\frac{Q_{i}}{B}$, and the production of each batch demands a time $T L_{i}$, The following constraints holds:

$$
\sum_{i=1}^{\mathrm{P}} \mathrm{Q}_{i} \mathrm{E}_{i} \leq \mathrm{H}
$$

The size of the storage tank $V T_{j}$, allocated after batch stage $\boldsymbol{j}$, is given by the following expression: ${ }^{25}$

$$
V T_{j} \geq S T_{i j}\left(B_{i j}+B_{i j+1}\right) \forall i=1, \ldots, M ; \forall j=1, \ldots, M-1
$$

Where $S T$ is the size factor corresponding to the intermediate storage tank, with identical definition to the batch stages. As no a priori tank allocation is given, binary variables $y_{j}$ are used to select their allocation. The value of variables $y$ is 1 if a tank is placed in position $j$, or zero otherwise. Cónstraint 12 is generalized to size the tank only if it exits:

$V T_{j} \geq S T_{i j}\left(B_{i j}+B_{i j+1}\right)-F_{j}\left(1-y_{j}\right) \forall i=1, \ldots, P ; \forall j=1, \ldots, M-1$

Where $F$ is a constant value sufficiently large such that when $y$ is 0 ( the tank does not exist), the constraint is trivially satisfied for any value of $V T_{j}$.

In particular, the cost minimization will drive $\mathrm{VT}=0$ . When the tank exists $\left(y_{j}=1\right)$ the term with $F_{j}$ vanishes, and the original constraint (12) holds. If the storage tank does not exist between two consecutive stages, then their batch sizes are constrained to be equal. Otherwise, this constraint is relaxed. This effect is imposed by the following constraints:

$1+\left(\frac{1}{\phi}-1\right) y_{j} \leq \frac{B_{i j}}{B_{i j+1}} \leq 1+(\phi-1) y_{j} \forall i=1, \ldots, P ; \forall j=1, \ldots, M-1$

Where $\Phi$ is a constant value corresponding to the maximum ratio allowed between two consecutive batch sizes.

In summary, the multiproduct plant design model that includes the options of parallel units in-phase and/or out of phase and provision of intermediate storage, consists of the objective function 5 subject to constraints $6,8,11,13$, and 14 , plus the upper and lower bounds that may apply. An important feature of the model is that both the objective function and the constraints are posynomial expressions that possess a unique local (and thus, global) solution. ${ }^{20}$ This basic model has been adapted to handle the particular feature of the composite stages (homogenizer, ultrafilters, and microfilters). In this case, constraint 6 is applied not to a general batch stage size but to each of the items that compose it. So in the case of microfilters, constraint 6 applies to both the retentate and the permeate vessels. A new parameter $S R$ was introduced to represent the size factor of the retentate vessel, ${ }^{i j}$ while $S_{i j}$ was left for the permeate vessel. Also in this case, the objective function must account for all the stage components. The notation $a$. and $\alpha$, were left for the cost coefficients of the permeate vessel, $b_{j}{ }_{j}$ and $\hat{\mathrm{a}}^{j}$ for the retentate vessel, and $d_{j}$ and $\gamma_{j}$ for the filtration area. A similar approach was implemented for the ultrafilters (retentate vessel and ultrafiltration area) and homogenizer (holding vessel and the homogenizer itself). 


\section{Methodology}

Between 1960s and 1970s witnessed a tremendous development in the size and complexity of industrial organizations. Administrative decision-making has become very complex and involves large numbers of workers, materials and equipment. A decision is a recommendation for the best design or operation in a given system or process engineering, so as to minimize the costs or maximize the gains. ${ }^{21}$ Using the term "best" implies that there is a choice or set of alternative strategies of action to make decisions. The term optimal is usually used to denote the maximum or minimum of the objective function and the overall process of maximizing or minimizing is called optimization. The optimization problems are not only in the design of industrial systems and services, but also apply in the manufacturing and operation of these systems once they are designed. Including various methods of optimization, we can mention: MINLP, Particle Swarm Optimization and Genetics Algorithms.

\section{Particle swarm algorithms}

The PSA is a population-based optimization algorithm, which was inspired by the social behavior of animals such as fish schooling and birds flocking, it can solve a variety of hard optimization problems. It can handle constrains with mixed variables requiring only a few parameters to be tuned, making it attractive from an implementation viewpoint. ${ }^{22}$ In PSA, its population is called a swarm and each individual is called a particle. Each particle flies through the problem space to search for optima. Each particle represents a potential solution of solution space, all particles form a swarm. The best position passed through by a flying particle is the optimal solution of this particle and is called pbest, and the best position passed through by a swarm is considered as optimal solution of the global and is called gbest. Each particle updates itself by pbest and gbest. A new generation is produced by this updating. The quality of a particle is evaluated by value the adaptability of an optimal function. In PSA, each particle can be regard as a point of solution space. Assume the number of particles in a group is $\mathrm{M}$, and the dimension of variable of a particle is N. The ith particle at iteration $\mathrm{k}$ has the following two attributes:

a. A current position in an $\mathrm{N}$-dimensional search space which represents a potential solution: $x^{k}=\left(x_{i, 1}^{k}, \ldots x_{i, n}^{k} \ldots x_{i, N}^{k}\right)$, where $\mathrm{x}_{\mathrm{i}, \mathrm{n}}^{\mathrm{k}} \varepsilon\left[\mathrm{I}_{\mathrm{n}}, \mathrm{u}_{\mathrm{n}}\right]$ is the $\mathrm{nth}$ dimensional variable, $1_{\mathrm{i}} n \mathrm{j} \mathrm{N}, l_{n}$ and $u_{n}$ are the lower and upper bounds for the nth dimension, respectively.

b. A current velocity, $v_{i}{ }^{k}=\left(v_{i, 1}^{k}, \ldots v_{i, n}^{k}, \ldots v_{i, N}^{k}\right)$, which controls its fly speed and direction. $V^{k}$ is restricted to a maximum velocity $\mathrm{v}_{\max }^{\mathrm{k}}=\left(\mathrm{v}_{\text {max }, 1}^{\mathrm{k}}, \ldots \mathrm{v}_{\max , \mathrm{n}}^{\mathrm{k}}, \ldots \mathrm{v}_{\max , \mathrm{N}}^{\mathrm{k}}\right)$. At each iteration, the swarm is uploaded by the following equations:

$$
\begin{gathered}
V_{i}^{k+1}=\omega V_{i}^{k}+c 1 r 1\left(P_{i}^{k}-X_{i}^{k}\right)+c 2 r 2\left(P_{g}^{k}-X_{i}^{k}\right) \\
X_{i}^{k+1}=X_{i}^{k}+V_{i}^{k+1}
\end{gathered}
$$

Where $P$ is the best previous position of the ith particle (also known as pbest) and $P$ is the global best position among all the particles in the swarm (also known as gbest). They are given by the following equations:

$$
P_{i}=\left\{\begin{array}{c}
P_{i}: f\left(\mathrm{X}_{i}\right) \geq P_{i} \\
X_{i}:: f\left(\mathrm{X}_{i}\right) \leq P_{i}
\end{array}\right.
$$

Where $f$ is the objective function, $M$ is the total number of particles. $r_{1}$ and $r_{2}$ are the elements generated from two uniform random sequences on the interval $[0,1]: \underset{1}{r} \alpha U(0,1) ; r_{2} \alpha U(0,1)$ and $\omega$ is an inertia weight ${ }^{30}$ which is typically chosen in the range of $[0,1]$. A larger inertia weight facilitates global exploration and a smaller inertia weight tends to facilitate local exploration to fine tune the current search area. Therefore the inertia weight $\omega$ is critical for the PSO's convergence behavior. A suitable value for the inertia weight $\omega$ usually provides balance between global and local exploration abilities and consequently results in a better optimum solution. Initially the inertia weight was kept constant. However, some literatures indicated that it is better to initially set the inertia to a large value, in order to promote global exploration of the search space, and gradually decrease it to get more refined solutions. $\mathrm{c}$ and $\mathrm{c}$ are acceleration constants which also control how far a particle will move in a single iteration.

\section{Genetic Algorithms}

GA, proposed in this paper based on the work of Wang et al., ${ }^{22}$ are related to the mechanics of natural selection and natural genetics. They combine the survival of the fittest among string structures with a structured yet randomized information exchange to form search algorithms with some of the innovative flair of human search. In every generation, a new set of individuals (strings) is created using bits and pieces of the fittest of the old individuals; while randomized, a GA are no simple random walk. They efficiently exploit historical information to speculate on new search points with expected improved performance. ${ }^{23}$ According to Wang et al. ${ }^{23}$ the canonical steps of the GA can be described as follows:

a. The problem to be addressed is defined and captured in an objective function that indicated the fitness of any potential solution.

A population of candidate solutions is initialized subject to certain constraints. Typically, each trial solution is coded as a vector $X$ , termed a chromosome, with elements being described as solutions represented by binary strings. The desired degree of precision would indicate the appropriate length of the binary coding.

Each chromosome $X, i=1,2, \ldots, P$, in the population is decoded into a form appropriate for evaluation and is then assigned a fitness score, $\mu(X)$ according to the objective.

Selection in genetics algorithms is often accomplished via differential reproduction according to fitness. In a typical approach, each chromosome is assigned a probability of reproduction, $P, i=1,2, \ldots, P$ , so that its likelihood of being selected is proportional to its fitness relative to the other chromosomes in the population. If the fitness of each chromosome is a strictly positive number to be maximized, this is often accomplished using roulette wheel selection (Goldberg, 1989). Successive trials are conducted in which a chromosome is selected, until all available positions are filled. Those chromosomes with above-average fitness will tend to generate more copies than those with below-average fitness. 
According to the assigned probabilities of reproduction, $\mathrm{P}, \mathrm{i}=1,2, \ldots, \mathrm{P}$, a new population of chromosomes is generated by probabilistically selecting strings from the current population. The selected chromosomes generate "offspring" via the use of specific genetic operators, such as crossover and bit mutation. Crossover is applied to two chromosomes (parents) and creates two new chromosomes (offspring) by selecting a random position along the coding and splicing the section that appears before the selected position in the first string with the section that appears after the selected position in the second string and vice versa. Bit mutation simply offers the chance to flip each bit in the coding of a new solution. According to our experiments, the parameters used for running GA and PSA are showed in Table 5.

Table 5 The parameters used for running GA and PSA

\begin{tabular}{lll}
\hline Algorithms & Parameters & Value \\
\hline & Population size & 200 \\
GA & Number of generations & 1000 \\
& Crossover probability & 0.6 \\
& Mutation probability & 0.4 \\
& Elitism & 1 \\
PSA & Number of particles & 200 \\
& Number of generations & 1000 \\
& Inertial weight & 1.00 \\
& Acceleration constants & 2.00
\end{tabular}

\section{Statistical analysis methods}

The interest in statistical analysis methods has grown recently in the field of computational intelligence. In this section, I will discuss the basic and give a survey of a complete set of variance analysis procedures developed to perform the comparison between PSA and GA, via the use of describing a test of the null hypothesis, which applies to independent random samples from two normal populations of size $n_{1}$ and $n_{2}$ are taken from normal population having the same variance, it follows $F$ distribution with $n_{1}-1$ and $n_{2}-1$ degrees of freedom, according to this equation:

$$
F=\frac{S_{1}^{2}}{S_{2}^{2}}
$$

However, The error from the optimal solution is given by:

$$
\text { error } \%=100 \frac{\left|\begin{array}{ll}
x_{\exp }-x_{\text {cal }}
\end{array}\right|}{x_{\exp }}
$$

In this research, $x \quad$ is considered to be the optimal solution founded by Montagna (Plant cost $\$ 829,500$ ), where the equation (19) is a criterion to confirm the optimal values.

\section{Results}

The problem could be formulated as the minimization of the investment cost for equipment and storage tanks. Given that the problem modeled has non linear objective function. For the purpose of optimization problem, the model developed has been solved with PSA and GAs Matlab Toolbox respectively, which is included in the GNU Octave Scientific Programming Language, using the data shown in Table 1-4. A horizon time of $6000 \mathrm{~h}$ has been considered. However, the intermediate storage cost coefficient with size factors is shown in Table 6.

Table 6 Intermediate storage cost coefficients and size factors

\begin{tabular}{lllll}
\hline \multirow{2}{*}{ Unit } & \multicolumn{5}{l}{$\mathbf{S T}_{i j}$ size factor for product $\mathbf{i}$ in stage $\mathbf{j}$} \\
\cline { 2 - 5 } Fermenter & Insulin & Vaccine & Chymosin & Protease \\
Microfilter I & 1.25 & 0.625 & 0.415 & 0.3125 \\
Homogenizer & 2.50 & 0.155 & 0.83 & 0.08 \\
Microfilter II & 2.50 & 0.155 & 0.83 & 0.08 \\
Ultrafilter I & 2.50 & 0.31 & 0.83 & 0.16 \\
Extractor & 0.40 & 0.20 & 0.135 & 0.10 \\
Ultrafilter II & 0.40 & 0.20 & 0.135 & 0.10 \\
Chromatography & 0 & 0 & 0.05 & 0.05 \\
\hline
\end{tabular}

On the other hand, the Table 7 shows the comparison of results for 30 runs between PSA and GA. 
Table 7 Comparison of results for 30 runs between PSA and GA

\begin{tabular}{lll}
\hline Values & PSA (\$) & GA (\$) \\
\hline Best & 912,450 & 833,674 \\
Average & 948,948 & $850,319.9401$ \\
Worst & $976,321.5001$ & $865,492.3154$ \\
Standart deviation & 9.7558 & 1.5327 \\
\hline
\end{tabular}

Nevertless, the optimization runs results for the investment cost calculated by PSA and GA during 30 runs is illustrated in Table 8 .

Table 8 Optimization runs results for the investment cost founded by PSA and GA during 30 runs

\begin{tabular}{llll}
\hline Technique & Plant cost (\$) & \%from optimal solución & CPU time (s) \\
\hline PSA & 912,450 & 10 & 800 \\
GA & 833,647 & 0.5 & 100 \\
\hline
\end{tabular}

Nonetheless, the equipment structure computed by PSA is showed in Table 9.

Table 9 Equipment structure calculated by PSA

\begin{tabular}{|c|c|c|c|c|c|c|c|c|}
\hline Stage & 1 & 2 & 3 & 4 & 5 & 6 & 7 & 8 \\
\hline$V_{j}$ & 24.7456 & $\frac{p: 2.1587}{r: 1.0794}$ & 1.1814 & $\frac{p: 2.3628}{r: 1.181}$ & 9.922 & 0.8921 & 0.6017 & 0.0825 \\
\hline$R_{j}$ & NA & A: 16.2041 & Cap: 1.0989 & A:8.668 & A: 109.3301 & NA & A: 17.8134 & NA \\
\hline$V T_{j}$ & 29.7066 & NA & NA & NA & 2.2154 & NA & 0.3795 & NA \\
\hline$M_{j}$ & 3 & 3 & 3 & 3 & 3 & 3 & 3 & 3 \\
\hline$G_{j}$ & 3 & 3 & 3 & 3 & 3 & 3 & 3 & 3 \\
\hline
\end{tabular}

However, Table 10 shows equipment structure calculated by GA.

Table I 0 Equipment structure calculated by GA

\begin{tabular}{|c|c|c|c|c|c|c|c|c|}
\hline Stage & I & 2 & 3 & 4 & 5 & 6 & 7 & 8 \\
\hline$V_{j}$ & 22.6085 & $\frac{p: 9.0651}{r: 4.5325}$ & I.0794 & $\frac{p: 2.1587}{r: 1.0794}$ & 9.0651 & 0.8151 & 0.5497 & 0.0754 \\
\hline$R_{j}$ & NA & A: I 4.8047 & Cap:I.004 & A:7.9194 & A: 99.8880 & NA & $A: 6.2750$ & NA \\
\hline$V T_{j}$ & 27.1410 & NA & NA & NA & $2.024 I$ & NA & 0.3795 & NA \\
\hline$G_{j}$ & I & I & I & I & I & I & I & I \\
\hline
\end{tabular}

The idle times in plant calculated by PSA is provided in Table 11. 
Table I I Idle times in plant calculated by PSA (seconds)

\begin{tabular}{lllllllll}
\hline Unit & & & & & & & & \\
\hline Product & I & 2 & 3 & 4 & 5 & 6 & 7 & 8 \\
Insulin & 0 & 0 & NA & NA & 0 & 57.7 & NA & 67.11 \\
Vaccine & 0 & 54 & 0 & 0 & 60.79 & 57.7 & 22.9 & 67.11 \\
Chymosin & 0 & 17 & NA & NA & 17.54 & 57.7 & 27.9 & 67.11 \\
Protease & 0 & 63 & 16 & I5 & 63.07 & 57.7 & 55.03 & 67.11 \\
\hline
\end{tabular}

However, the idle times in plant calculated by GA is shown in Table 12.

Table I 2 Idle times in plant calculated by GA (seconds)

\begin{tabular}{lllllllll} 
Unit & & & & & & & & \\
Product & $\mathrm{I}$ & 2 & 3 & 4 & 5 & 6 & 7 & 8 \\
Insulin & 0 & 0 & NA & NA & 0 & 0.01 & 0 & 0 \\
Vaccine & 0 & 1.93 & 0.04 & 0 & 2.91 & 0 & 0.17 & 0 \\
Chymosin & 0 & 0.01 & NA & NA & 0 & 0 & 0.31 & 0.17 \\
Protease & 0 & 2.09 & 0 & 0 & 3.07 & 0 & 0.5 & 0 \\
\hline
\end{tabular}

The results of the statistical analysis are illustrated in Table $13 \&$ Table 14 .

Table I 3 The results of two algorithms solving MBPD problem

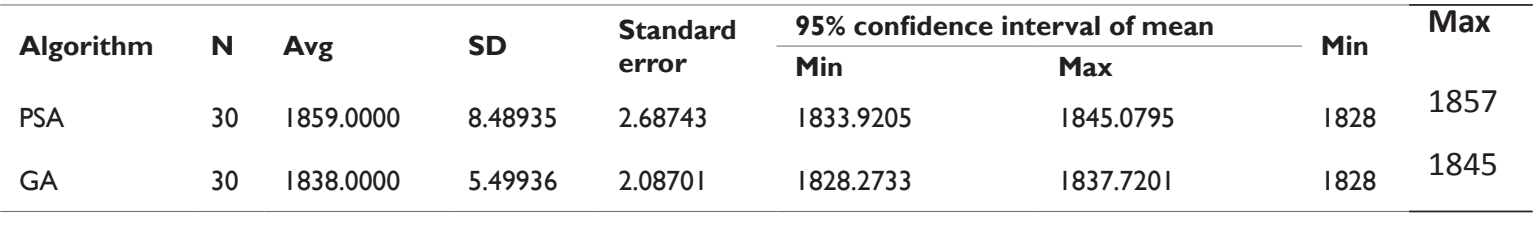

Table I4 Variance analysis result of MBPD problem

\begin{tabular}{llllll}
\hline & Quadratic sum & Free degree & $\begin{array}{l}\text { Mean } \\
\text { Square }\end{array}$ & F & Significance \\
\hline SDB & 2339.676 & 3 & 779.895 & 15.455 & 0.000 \\
SDI & 1814.100 & 36 & 50.392 & - & - \\
SUM & 4154.775 & 39 & - & - & - \\
\hline
\end{tabular}

\section{Discussion}

It is clear from the summary of the results shown in Table 7, that the performance of both PSA and GA produce adequate values regarding the cost for equipment and storage tanks. However, GA performs better than the PSA in terms of the average and the worst fitness values and the standard deviation. Table 7, also, shows the best final solution found in the 30 runs of PSA and GA. According to our knowledge, the case study about the optimal design of protein production plant has been taken from Montagna. However, they solved the problem using rigorous mathematical programing (MINLP), their model includes 104 binary variables and has been convexified using the transformation proposed by Kocis and Grossmann. The MINLP model has been solved using DICOPT ++ , which is included in the GAMS optimization modeling software. The algorithm implemented in DICOPT ++ relies on the Outer Approximation/Equality Relaxation/Augmented Penalty (OA/ER/AP) method. The OA/ER/AP solution method consists of the decomposition of the original MINLP problems into a sequence of two subproblems: a non linear programming (NLP) subproblem and a mixed integer linear programming (MILP) subproblem also known as the Master problem, which is solved to global optimality (minimize the caplital cost $\$ 829,500)$. However, in previous work of Montagna and other, their model needed a long computational time (more than 86400 seconds) and require several initial values to the optimization variables, they also showed in their paper that the behavior of the demand was completely deterministic.

Whilst, this assumption does not seem to be always a reliable representation of the reality, since in practice the demand of pharmaceutical products resulting from the batch industry is usually variable. Simulations outcomes were then compared with experimental data in order to check the accuracy of the method. Table 8 presents the results obtained in different optimization runs for multiproduct batch plant design. For each simulator run, the average numerical effort spent on solving the problem on LINUX System, Intel ${ }^{\circledR} D$, CPU2.80 GHz, 2.99 of RAM. Table 8 shows plant cost, \% from optimal solution and CPU time obtaining during 30 runs. PSA and GA performed effectively and give a solution within 10 and $0.5 \%$ of the global optimal $\$ 912,450$ and $\$ 833,647$, respectively. Furthermore, the important feedback could be taken from Table 8 , is the GA results in a faster convergence than PSA and the MINLP algorithm. In addition, the GA is so close to the global optimal of MBPD $(0.5 \%$ from optimal solution) and provides also an interesting solution, in terms of quality as well as of computational time as illustrated in Table 8, while Table 9 presents the sizes for the units involving a set of discrete equipment structure given by PSA. The inconvenience of this configuration is just stopped at $6000 \mathrm{~h}$ with risk of failing to fulfill the potential future demand coming from a fluctuation of the market. 
In order to show how the evolution process is going on for both PSA and GAs, respectively, the convergence of the best fitness values. The convergence rate of objective function values as a function of generations for both PSA and GAs where for clarity only 1000 generations are shown. For the optimization problem considered, GAs decrease rapidly and converge at a faster rate (around 500 generations) compared to that for PSA (about 800 generations), from which it is clear that GAs seem to perform better compared to PSA. So, for the present problem the performance of the GAs is better than PSA from an evolutionary point of view.

To compare the computational time, the swarm/population size is fixed to 200 for both PSA and GAs algorithms. Whereas, the generation number is varied. Simulation were carried out and conducted on LINUX System, Intel (R) D, CPU 2.80GHz, 2.99 of RAM Computer, in the GNU Octave environment. Here the result in the form of graph is shown in. It is clear from that the computational time for GAs is very low compared to the PSA optimization algorithm. Further, it can also be observed from hat in case of GAs the computational time increases linearly with the number of generations, whereas for PSA the computational time increases almost exponentially with the number of generations. The higher computational time for PSA is due to the communication between the particles after each generation. Hence as the number of generations increases, the computational time increases almost exponentially.

Table 9 presents the sizes for the units involving a set of discrete equipment structure given by PSA. The inconvenience of this configuration is just stopped at 6000 hours with risk of failing to fulfill the potential future demand coming from a fluctuation changing of the market.

On the other hand, the calculation of the structure of equipment using GA is illustrated in Table 10. The total production time, also, computed by GA is 5491.12 hours to fulfill the eventual increase of future demand caused by market fluctuation. In addition, the GAs results in a faster convergence. However, the equipment structure showed by PSA is very expensive. Furthermore, the PSA approach has the disadvantage of long CPU time.

At the same time as, the GA allow the reduction of the idle time to the stage, in any way, Table $11 \&$ Table 12 show the idle times obtained by PSA and GA respectively.

However, some observations about some important aspects in our implication of GAs and some problems in practice: the most important of all is the method of coding, because the codification is very important issue when a genetic algorithm is designed to dealing with combinatorial problem, also of the characteristics and inner structure of the DMBP.

The commonly adopter concatenated, multi-parameter, mapped, fixed point coding are not effective in searching for the global optimum. According to the inner structure of the design problem of multiproduct batch that gives us some clues for designing the above mixed continuous discrete coding method with a four-point crossover operator. As is evident from the results of application, this coding method is well fit for the proposed problem.

Another aspect that affects the effectiveness of our Genetic Algorithms procedure considerably is crossover.

Corresponding to the proposed coding method, we adopted a fourpoint crossover. It is commonly believed that multipoint crossover is more effective than the traditional one point crossover method.
It is also important to note that the selection of crossover points as well as the way to carry out the crossover should take in account the bit string structure, as is the case in our codification.

One problem in practice is the premature loss of diversity in the population, which results in premature convergence, because premature convergence is so often the case in the implementation of GA according to our calculation experience.

Our experience makes it clear that the Elitism parameter can solve the premature problem effectively and conveniently.

In order to further explain the effects of these algorithms on solving the MBPD problem, the variance analysis was performed. Each of the PSA and GA algorithms was run 30 times. The Minitab software was used to analyze the results. Therefore, the results are given in Table 13 \& Table 14

Table 14 indicates that, the mean square deviation between groups (SDB) is 779.895 . The mean square deviation within groups (SDI) is 50.392. The test statistic $\mathrm{F}=15.477$. If significance level $\alpha=0.05$, then the critical value $2.92 \leq \mathrm{F}_{\alpha}(3.36) \leq 2.84$. Thus, $\mathrm{F}>\mathrm{F}_{\alpha}$ (3.36) indicating that the difference between the average is significant, that is, the performance difference of algorithms is significant.

Nevertheless, these techniques are not a panacea, despite their apparent robustness, there are control "parameters" involved in these metaheuristics and appropriate setting of these parameters is a key point for success.

\section{Conclusion}

Techniques such as PSA and GA are inspired by nature, and have proved themselves to be effective solutions to optimization problems. We applied Genetic Algorithms with an effective mixed continues discrete coding method with a four crossover point to solve the problem of DMBP. GA perform effectively and give a solution within $0.5 \%$ of the global optimum. Whilst, it is observed that, in terms of computational time, the GAs approach is faster. The computational time increases linearly with the number of generations for GA, whereas for PSA the computational time increases almost exponentially with the number of generations, interpreting that, the higher computational time for PSA is due to the communication between the particles after each generation. Furthermore, the results provided by GA are much better with respect to PSA. In this paper, the GA gave us the highest efficiency and justifies its use for solving nonlinear mathematical models. Therefore, this work provides an interesting decision/making approach to improve the design of multiproduct batch plants under conflicting goals.

\section{Acknowledgements}

The authors Dr. Youness El Hamzaoui and Dr. Juan Antonio Alvarez Arellano from Facultad de Ingenieria, Universidad Autonoma del Carmen, expresse their gratitude to Dr. Jose Alfredo Hernandez Perez from CIICAP-UAEM for his comments and suggestions in order to improve this research article.

\section{Conflict of interest}

The author declares that their is no conflict of interest.

\section{References}

1. Dietz A, Azzaro-Pantel C, Pibouleau L, et al. Multiobjective optimization for multiproduct batch plant design under economic and environmental 
considerations. Computers \& Chemical Engineering. 2006;30(4):599_ 613.

2. Dietz A, Azzaro-Pantel C, Pibouleau L, et al. Strategies for multiobjective genetic algorithm development: application to optimal batch plant design in process systems engineering. Computers \& Industrial Engineering. 2008;54(3):539-569.

3. Ponsich A, Azzaro-Pantel C, Domenech S, et al. Mixed-integer nonlinear programming optimization strategies for batch plant design problems. Industrial \& Engineering Chemistry Research. 2007;46(3):854-863.

4. Dietz A, Aguilar-Lasserre A, Azzaro-Pantel C, et al. A fuzzy multiobjective algorithm for multiproduct batch plant: application to protein production. Computers \& Chemical Engineering. 2008;32(1-2):292-306.

5. Aguilar-Lasserre AA, Bautista Bautista MA, Ponsich A, et al. An AHPbased decision-making tool for the solution of multiproduct batch plant design problema under imprecise demand. Computers \& Operations Research. 2009;36:711-736.

6. Aguilar-Lasserre A, Giner AH, Azzaro-Pantel C, et al. Multiobjective multiproduct batch plant design under uncertainty: application to protein production. 10th International Symposium on Process Systems Engineering. Brazil: Salvador; 2009.

7. Borisenko A, Kegel P, Gorlatch S. Optimal design of multi-product batch plants using a parallel branch-and-bound method. In: Malyshkin V, editor. Berlin: Springer; 2011:417-430.

8. Hasebe S. Optimal scheduling and minimum storage tank capacities in a process system with parallel batch units. Computers \& Chemical Engineering. 1998;3(4):185-195.

9. Cao DM, Yuan XG. Optimal design of batch plants with uncertain demands considering switch over of operating modes of parallel units. Industrial \& Engineering Chemistry Research. 2002;41(18):4616-4625.

10. Crougham M, Caldwell V, Randlev B, et al. Prediction of Culture Performance through Cell Cycle Analysis: A Potential Tool in Operations Scheduling. Presented at the Biochemical Engineering X Conference. Canada; 1997.

11. Montagna JM, Iribarren OA, Galiano FC. The design of multiproduct batch plans with process performance models. Chemical Engineering Research and Design. 1994 :783.
12. Azzaro-Pantel C, Dietz A, Pibouleau L, et al. A framework for multiproduct batch plant design with environmental consideration: application to protein production. Industrial \& Engineering Chemistry Research. 2005;44(7):2191-2206.

13. Dessouky Y, Bayer A. A simulation and design of experiment modeling approach to analyze facility maintenance costs. Computer \& Industrial Engineering. 2002;43(3):423-436.

14. Montagna JM, Vecchietti AR, Iribarren OA, et al. Optimal design of protein production plants with time and size factor process models. Biotechnol Prog. 2000;16:228-237.

15. Birattari M, Zlochin M, Dorigo M. Towards a theory of practice in metaheuristics design: a machine learning perspective. Theoretical Informatics and Applications. 2006;40:53-369.

16. Petrides D, Sapidou E, Calandranis J. Computer aided process analysis and economic evaluation. Biotechnol Bioeng. 1995 ;48(5):529-5415.

17. Asenjo JA, I Patrick. Large Scale Protein Purification. In: E Harris, Angal S, editors. Protein Purification Applications: A Practical Approach. IRL Press: USA; 1990.

18. Salomone HE, Iribarren OA. Posynomial modeling of batch plants: A procedure to include process decision variables. Computers \& Chemical Engineering. 1992;16:173-184.

19. Mistry SL, Kaul A, Merchuk JC, et al. Mathematical modeling and computer simulation of aqueous two phase continuous protein extraction. Journal of Chromatography A. 1996;741:151-163.

20. Modi AK, Karimi IA. Design of multiproduct batch processes with finite intermediate storage. Computers \& Chemical Engineering. 1989;13:127139

21. Grossmann IE, Sargent RW. Optimal design of multipurpose chemical plants. Industrial Engineering Chemical Process. 1979;18(2):343-348.

22. Shi Y, Eberhart RA. Modified particle swarm optimizer. IEEE Proceeding. 1998:69-73.

23. Wang C, Quan H, Xu X. Optimal design of multiproduct batch chemical process using genetic algorithm. Industrial \& Engineering Chemistry Research . 1996;35(10) :3560-3566. 